\title{
Microwave-assisted xylanase reaction: the impact in the production of prebiotic xylooligosaccharides
}

\author{
Hugo Mobarec ${ }^{1,3}$, Rodrigo Villagomez ${ }^{2}$, Eva Nordberg Karlsson ${ }^{1}$, \\ Javier A. Linares-Pastén ${ }^{1, *}$
}

${ }^{1}$ Lund University, Division of Biotechnology, Department of Chemistry, Lund, Sweden

${ }^{2}$ Lund University, Centre for Analysis and Synthesis, Lund University, Lund, Sweden

${ }^{3}$ Technical University of Denmark, Department of Chemical and Biochemical Engineering, Copenhagen, Denmark

*Corresponding authors: J. A. Linares-Pastén

(e-mail: javier.linares_pasten@biotek.lu.se) 


\section{Abstract}

2

3 The enzymatic production of prebiotic xylooligosaccharides (XOS) has become an 4 attractive way to valorize lignocellulosic biomass. However, despite numerous xylanases 5 reported for potential use in the production of XOS, most of the family GH10 also 6 produce xylose. This monosaccharide can negatively affect the selectivity to stimulate 7 the growth of intestinal microorganisms beneficial to human health. In this work, a 8 thermostable alkali-tolerant xylanase (BhXyn10A) from Bacillus halodurans $\mathrm{S} 7$ has been 9 used to produce XOS under conventional convective heat transfer and microwave 10 radiation. The microwave-assisted reaction markedly decreases the xylose content in the 11 hydrolysates and significantly increases the yield of XOS, compared to conventional 12 heating. Molecular dynamic simulations of BhXyn10A have shown an increased 13 fluctuation of the amino acids of the aglycone subsites suggesting that these subsites can 14 determine the production of xylose. Thus, the microwave heating could affect the amino 15 acid fluctuations in the aglycone subsites reducing the xylose formation. These findings 16 open up new avenues in enzyme technology for the production of XOS. 


\section{Introduction}

19 Lignocellulosic biomass has been increasingly gaining attention because of its potential as a raw material for the biochemical industry in a biorefinery perspective. After a pretreatment stage, lignocellulosic biomass can be fractionated into its three main components - cellulose, hemicellulose and lignin -from which each one can be subsequently processed separately for manufacturing a wide variety of products. For instance, hemicelluloses can be transformed into a number of molecules such as xylitol, erythitol, ferulic acid, furfural, ethanol, lactic acid and xylooligosaccharides (XOS). ${ }^{1}$

Particular interest has been put into the production of XOS, they can selectively stimulate the growth of probiotic bifidogenic and lactic acid bacteria residing in the human gut.$^{2}$ Nonetheless, it has also been found that it is mostly XOS with a degree of polymerization (DP) from 2 to 4 that selectively induce the growth of beneficial microorganisms in the human digestive tract. ${ }^{2}$ Therefore, the xylose (DP1) content should be minimized to enhance the quality of the prebiotic mixture.

The hydrolysis of xylan-rich hemicelluloses into XOS can be attained by enzymatic conversion. Xylanases can be either endo-acting (EC. 3.2.1.8) or exo-acting enzymes (EC. 3.2.1.156 and 3.2.1.37). Since exo-acting xylanases release xylose monomers (DP1), only endo-acting xylanases are of interest for XOS production., ${ }^{3,4}$

It has been found that the composition profile of the products after the xylanase-mediated hydrolysis significantly differs according to the enzyme used, reaction conditions, such as time, temperature and substrate. ${ }^{5}$ Henceforth, optimizing both the proportion of XOS with oligomers (DP2-4) in the mixture and the hydrolysis yield will be key factors for designing an effective production process. Additionally, it has been suggested that microwave radiation might have a synergic effect in combination with enzyme catalysis, favoring both the yield and the product selectivity. ${ }^{6}$

Thermostable enzymes are attractive biocatalysts in a variety of biorefinery processes, ${ }^{7}$ due to their stability and capacity to perform reactions in high temperatures. At these conditions, polymeric substrates, such as xylan, are more soluble, making the reaction mixture lower viscous, increasing the yield and the efficiency of the process. Besides, 
these enzymes can be coupled to microwave reactors due to their thermostability, allowing the development of green-chemistry processes for the manufacture of novel products.

The objective of this work was to investigate the effect of microwave heating in the enzymatic production of XOS. Three variants of a thermostable alkali-tolerant xylanase from Alkalihalobacillus halodurans (Bacillus halodurans S7) ${ }^{8}$ were studied: (1) BhXyn10A, is the wild type (wt) form, (2) BhXyn10K80R, is a mutant that has shown a higher activity than $w t$ under conventional conditions, ${ }^{1}$ and (3) BhHXyn10A, is a variant containing an additional $\mathrm{N}$-terminal tail. ${ }^{9}$ Birchwood xylan was used as a substrate. Besides, ligand/enzyme docking, and molecular dynamics simulations were performed to study the structural implications in the production of XOS and xylose.

\section{Material and Methods}

\subsection{Chemicals.}

Xylan from, birchwood, beechwood and larchwood were purchased from Sigma Aldrich (Saint Louis, Missouri). Xylan from quinoa (Chenopodium quinoa) stalks was extracted as it is described in our previous work. ${ }^{10}$ Analytical grade xylose, xylobiose, xylotriose, xylotetrose, xylopentose and xylohexose were obtained from Megazyme (Wicklow, Ireland).

\subsection{Enzymes: production and purification}

Production. BhXyn10AH, BhXyn10K80R and BhHXyn10A enzymes encoded by a synthetic gene (GeneBank accesion number: MW311490) were produced in Escherichia coli BL21(DE3) in 2.5 L bioreactors as described in our previous work. ${ }^{11}$ Pre-inoculums were prepared in $100 \mathrm{~mL}$ of mAT medium ${ }^{12}$ with $10 \mathrm{~g} / \mathrm{L}$ glucose as sole carbon source. E. coli strains harboring plasmids pET21::BhXyn10AH, and pET21::BhXyn10AK80R were inoculated in culture media supplemented with $100 \mu \mathrm{g} / \mathrm{mL}$ ampicillin, while the medium for the strain $E$. coli 21(DE3) pET28::BhHXyn10A was supplemented with 34 $\mu \mathrm{g} / \mathrm{mL}$ kanamycin. All pre-inoculums were growth during 12 hours in shake flasks at $30^{\circ} \mathrm{C}$. Reactors containing $2.4 \mathrm{~L} \mathrm{mAT}$ medium and set up at $37^{\circ} \mathrm{C}$ and $40 \% \mathrm{O}_{2}$ saturation, 
were inculcated with $100 \mathrm{~mL}$ of inoculums previously described. Recombinant protein expression was induced with $1 \mathrm{mM}$ isopropyl- $\beta$-d-thiogalactopyranoside (IPTG) when the optical density of the cultivations reached 3 at $600 \mathrm{~nm}$. The induction period was for 2 hours. Cell pellets were harvested by centrifugation (4500 g), $10 \mathrm{~min}$, for protein purification, while the supernatants were discarded.

Purification. Cell pellets were resuspended in binding buffer consisting in $20 \mathrm{mM}$ Tris$\mathrm{HCl}, 0.5 \mathrm{M} \mathrm{NaCl}, \mathrm{pH}=7.4$; and lysed by ultrasonication in intervals of $10 \times 10 \mathrm{~min}$. Next, lysates were centrifuged at $5500 \mathrm{~g}$ for $20 \mathrm{~min}$, pellets were discarded, and supernatants were used for the protein purifications by immobilized metal ion affinity chromatography (IMAC). A ÄKTA prime system (Amersham Biosciences, Sweden) with a $5 \mathrm{~mL}$ FF HiTrap nickel column (GE Health Care, Germany) was used. The method was: column equilibration with 5 volumes of binding buffer, injection of samples (supernatants) through the system, washing with binding buffer until reach the equilibration-baseline absorbance and elution with linear gradient of buffer $20 \mathrm{mM}$ Tris- $\mathrm{HCl}, 0.5 \mathrm{M} \mathrm{NaCl}, 0.5$ $\mathrm{M}$ imidazole, $\mathrm{pH}=7.4$. Purity and concentration of the purified proteins were analyzed by SDS-PAGE and spectrophotometry respectively.

\subsection{Enzymatic reactions}

\subsubsection{Conventional heating reactions}

Four types of xylan, from birchwood, beechwood, larchwood and quinoa stalks, were used as substrates for three variants (BhXyn10AH, BhXyn10K80R and BhHXyn10A) of the alkali-tolerant Bacillus halodurans S7 endoxylanase, giving in total twelve different reactions. In addition, every reaction was performed in triplicate, as well as the corresponding controls without enzyme. Every reaction was prepared in $200 \mu \mathrm{L}$, containing, 1\% xylan, $100 \mathrm{mM}$ glycine- $\mathrm{NaOH}$ buffer $\mathrm{pH}=9$ and $6.67 \mathrm{mg} / \mathrm{L}$ of enzyme. The reactions were incubated at $62{ }^{\circ} \mathrm{C}$ during $15 \mathrm{~h}$. The reactions were started by adding the enzyme and stopped heating at $100{ }^{\circ} \mathrm{C}$ for $10 \mathrm{~min}$.

\subsubsection{Microwave assisted reactions}


118 Xylan from birchwood and quinoa stalks were used as substrates. Every reaction mix was

119 prepared up to a final volume of $6 \mathrm{~mL}$, in $20 \mathrm{ml}$ microwave vials provided with a magnet

120 for mixing. The components were $1 \%$ xylan, $100 \mathrm{mM}$ glycine- $\mathrm{NaOH}$ buffer $\mathrm{pH}=9$ and

$1216.67 \mathrm{mg} / \mathrm{L}$ of enzyme. The reactions were heated in an Initiator+ Microwave Synthesizer

122 (Biotage, Sweden) reactor. The programs consisted in varying the reaction time in the

123 range from 0 to 30 minutes at $62{ }^{\circ} \mathrm{C}$ followed by an enzyme deactivation stage of $10 \mathrm{~min}$

124 at $100{ }^{\circ} \mathrm{C}$. The power input oscillated between 0 and $40 \mathrm{~W} / \mathrm{g}$ of reaction medium.

\subsection{Temperature profiles}

128 Xylanase BhXyn10AH activity was determined in a range of temperatures from 40 to 85

$129{ }^{\circ} \mathrm{C}$, both in conventional as well as microwave heated reactions. The reaction mixtures 130 were prepared up to a final volume of $6 \mathrm{~mL}$, containing $1 \%$ birchwood xylan, $50 \mathrm{mM}$ 131 glycine buffer $\mathrm{pH}=9$ and $6.67 \mathrm{mg} / \mathrm{L}$ of enzyme. Activities were quantified after $10 \mathrm{~min}$ 132 of incubation by DNS method for reducing end sugars such is described below (section 2.5.2).

2.5. Analytical methods

\subsubsection{Protein analysis}

Recombinant protein expression and purified fractions were analyzed by SDS-PAGE.

140 The concentration of the purified enzymes was determined in a NanoDrop (Thermo

141 Fisher Scientific, United States of America) and corroborated by spectrophotometry at $280 \mathrm{~nm}$ of wavelength.

\subsubsection{Enzyme activity analysis}

146 Products of the enzymatic reactions, both under conventional and microwave heating, were analyzed by a colorimetric assay, using 3,5-dinitrosalicylic acid (DNS) for quantifying reducing sugars. The DNS solution contained a 1:1:1:1 volumetric mixture of $1 \%$ DNS, $40 \%$ Rochelle salt, $0.2 \%$ phenol, $0.5 \%$ potassium disulfide, and all these

150 components were mixed in $1.5 \%$ sodium hydroxide. A volume of $400 \mu \mathrm{L}$ of the 151 hydrolysate was mixed with $600 \mu \mathrm{L}$ of DNS solution and heated to $100^{\circ} \mathrm{C}$ for 10 minutes. 
152 Subsequently, the obtained colored solution was diluted 10 times and then transferred to 153 a microplate well for its quantification at $540 \mathrm{~nm}$ in a microplate spectrophotometer 154 (Thermo Scientific ${ }^{\mathrm{TM}}$, Multskan ${ }^{\mathrm{TM}} \mathrm{GO}$ ). The actual concentrations were calculated based

155 on a calibration curve elaborated with xylose in a range of 0 to $2 \mu \mathrm{mol}{ }^{1}$

2.5.3. Oligosaccharide analysis

159 Oligosaccharides produced both in conventional and microwave assisted reactions were analyzed by high-performance anion-exchange chromatography with pulsed amperometric detection (HPAEC-PAD). A Dionex chromatography system in combination with a PA-100 column (Thermo Fisher Scientific, United States of America) was used, such is described in our previous work. ${ }^{9}$

\subsection{Computational studies}

2.6.1. Construction of the complex enzyme / ligands

The following model of a complex between the enzyme and ligands (products of hydrolysis) was build: BhXyn10AH / $\beta$-d-Xylp-(1-4)- $\beta$-d-Xylp-(1-4)- $\beta$-d-Xylp / $\beta$-dXylp-(1-4)- $\beta-d-X y l p$. The atomic coordinates of the ligands were transferred from the crystallographic structure of the complex Streptomyces olivaceoviridis E-86 (PDB: 1ISX) to the active site of the crystallographic structure of the apoenzyme BhXyn10AH (PDB: 2UWF), by superposition of both structures using CHIMERA v1.14.. ${ }^{13}$ Thereafter, the reducing-end xylose of the xylotriose located in the aglycone side was removed. The obtained complex was energetically minimized using YASARA v18.4.24 ${ }^{14}$ with the AMBER14 force field. ${ }^{15}$

2.6.2. Molecular dynamic simulations

The minimized complex was subjected to molecular dynamics simulations with explicit molecules of water as solvent. All calculations were performed in YASARA v18.4.24, ${ }^{14}$ using AMBER14 force field. ${ }^{15}$ A cubic simulation cell, $20 \AA$ larger than the complex, with periodic boundary conditions, was filled with TIP3P water molecules and counter ions. ${ }^{16}$ The distance for Van der Waals interactions was set in a medium range of $8 \AA$ and 
186 the long-range Coulomb forces were calculated using the particle-mesh Ewald 187 algorithm. ${ }^{17}$ The temperature control was through the Berendsen Thermostat. ${ }^{18}$ The 188 simulated conditions were: $0.9 \% \mathrm{NaCl}, \mathrm{pH} 9,0.982 \mathrm{~g} / \mathrm{mL}$ solvent density and $335{ }^{\circ} \mathrm{K}$ 189 during 50 ns, saving snapshots every 100 ps. Root-mean-square deviations (RMSD) of 190 atomic coordinates, root-mean-square fluctuations (RMSF) and enzyme / ligand 191 interactions were analyzed.

192

\section{Results and discussion}

\subsection{Enzymes production}

Three variants (BhXyn10AH, BhXyn10K80R and BhHXyn10A) of the 1,4- $\beta$-endoxylanase from Bacillus halodurans S7 were successfully produced in of E. coli BL21(DE3) harboring synthetic genes. BhXyn10A is the wild type form with a histidine tag in the C-terminal; BhXyn10-K80R has an arginine residue instead of lysine in the position 80 . The form $B h \mathrm{HXyn} 10 \mathrm{~A}$ has an additional tail of 16 amino acids and a histidine tag in the N-terminal. The recombinant strains were cultivated in a batch reactor $(2.5 \mathrm{~L})$ using the synthetic medium NYAT with $10 \mathrm{~g} / \mathrm{L}$ of glucose (S) as sole carbon source. High productivity (Table 1) of soluble recombinant protein were reached after $5 \mathrm{~h}$ of cultivation, from which the last $2 \mathrm{~h}$ were the production phase initiated with the addition of inducer (IPTG).

Table 1. Yield coefficients, $Y_{\mathrm{P} / \mathrm{S}}$ (mass of recombinant protein/mass of substrate), $\mathrm{Y}_{\mathrm{P} / \mathrm{X}}$ (mass of recombinant protein/biomass) and volumetric productivity $\left(\mathrm{Q}_{\mathrm{P}}\right)$ of recombinant variants of 1,4- $\beta$-endo-xylanase from Bacillus halodurans $\mathrm{S} 7$ in E. coli BL21(DE3) in

211 batch cultivations.

\begin{tabular}{cccc}
\hline Productivity & BhHXyn10A & BhXyn10AH & BhXyn10K80R \\
\hline $\mathrm{Y}_{\mathrm{P} / \mathrm{S}}(\mathrm{g} / \mathrm{g})$ & 0.04 & 0.13 & 0.04 \\
$\mathrm{Y}_{\mathrm{P} / \mathrm{X}}(\mathrm{g} / \mathrm{g})$ & 0.06 & 0.26 & 0.12 \\
$\mathrm{Q}_{(\mathrm{P})}\left(\mathrm{g} \mathrm{L}^{-1} \mathrm{~h}^{-1}\right)$ & 0.04 & 0.15 & 0.05 \\
\hline
\end{tabular}

\subsection{Production of XOS in conventional reactions}

215 Xylan from birchwood, beechwood, larchwood and quinoa stalks were used as substrates 216 for each variant of the 1,4- $\beta$-endoxylanase from B. halodurans produced (Figure 1).

217 BhXyn10AH and BhXyn10K80R have shown similar product profiles on all the 
218 substrates used, being the main product xylobiose (X2) xylotriose (X3) and xylose (X).

219 However, the mutant K80R have yielded slightly higher amount of the products 220 mentioned. This result is even more pronounced when the substrate is xylan from quinoa

221 (Figure 1). These results are consistent with the higher activity of the mutant respect to 222 the wild type, such as was reported previously, ${ }^{1}$ and with the slightly higher yield of XOS 223 obtained from quinoa xylan using the mutant K80R.

On the other hand, the variant BhHXyn10A produced lower total amount of XOS (lower than $20 \mathrm{mg}$ XOS per $1 \mathrm{~g}$ of xylan). However, interestingly, its product profiles were different to the other two variants, since relatively long XOS, such as xylotetraose (X4), xylopenaose (X5) and xylohexaose (X6), were produced in similar amounts as X2 and X3 (Figure 1). This type of products profiles was obtained initially for the hydrolysis of quinoa xylan, and later in this work for the hydrolysis of beechwood, birchwood and largewood xylans. Thus, the elongation of the $\mathrm{N}$-terminal tail in the variant $B h \mathrm{HXyn} 10 \mathrm{~A}$ affected significantly both the yield and the product profile.

BhXyn10AH

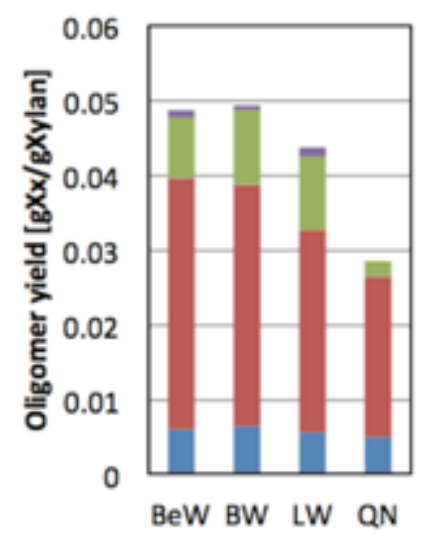

$\mathrm{x}_{1}=\mathrm{X}_{2}=\mathrm{X}_{3}=\mathrm{X}_{4}=\mathrm{X}_{5}=\mathrm{X}_{6}$
BhXYn10AK80R

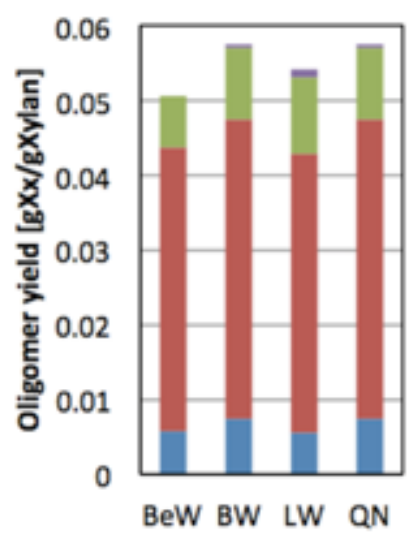

$\mathbf{E} \times 1=\times 2=\times 3=\times 4=\times 5=\times 6$
BhHXyn10A

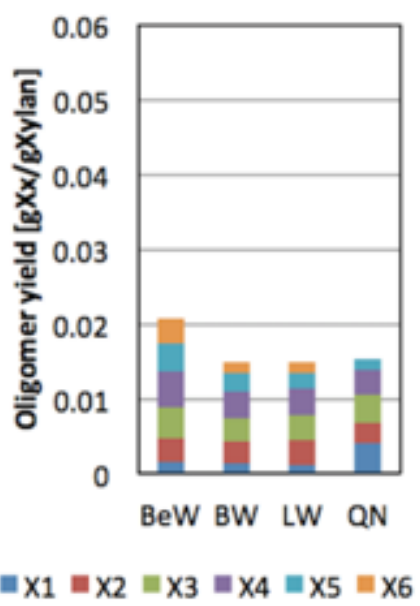

Figure 1. Product profiles of the enzymatic conversion of different xylans to xylose and XOS under conventional heating. Substrates were: BeW (beechwood xylan), BW (birchwood xylan), LW (larchwood xylan) and QN (quinoa xylan). Analyzed products were X1 (xylose), X2 (xylobiose), X3 (xylotriose), X4 (xylotetrose), X5 (xylopentose) and X6 (xylohexose). Variants BhXyn10AH, BhXyn10K80R and BhHXyn10A of Bacillus halodurans 1,4- $\beta$-endoxylanase were used. 
244 The monosaccharide xylose was produced in all of the reactions, independently of the 245 substrate type or endoxylanase variant. However, xylose is an undesirable co-product 246 since it does not selectively promote the probiotic growth as XOS do. Indeed, 247 endoxylanases with low rate of xylose production are preferred in the prebiotic 248 development. Microwave-assisted reactions have shown to increase the yield and 249 specificity of reactions catalyzed both enzymatic and no-enzymatic catalyzers ${ }^{19}$ 250 Therefore, we have hypothesized that the microwave-assisted endoxylanase reaction can 251 reduce the co-production of xylose and enhance the production of XOS.

253 Microwave pretreatment of xylan has shown to improve its subsequent enzymatic 254 hydrolysis ${ }^{20}$ under conventional heating. However, in the best of our knowledge, the 255 effect of the microwave irradiation in the endoxylanase activity has not been reported until this work. It was found that the microwave assisted enzymatic reaction gave significantly higher activity than the conventional heated reaction (Table 2).

\subsection{Production of XOS in microwave-assisted reactions}

260

261 Initially, the reaction time and optimal temperature were determined quantifying the total 262 reducing end sugars (DNS assay) produced enzymatically from birchwood xylan. The 263 reaction course was monitored during 30 minutes at $62^{\circ} \mathrm{C}$ and $\mathrm{pH}=9$. It can be 264 appreciated that at these conditions, even after some seconds of reaction, a detectable 265 amount of product is obtained (Figure 2A). This observation is valid for enzymes 266 BhXyn10A and BhXyn10AK80R. Nonetheless, no activity was detected for the variant 267 BhHXyn10A. Since the other two variants appear to be working efficiently, denaturation 268 might not be a likely explanation. It could be that the added tag (16 amino acids including 269 the histidine tag) attached to the N-terminus, under microwave radiation gets oriented in 270 such a way that interferes with the active site of the enzyme. But further research is needed 271 to confirm this claim. 
(A)

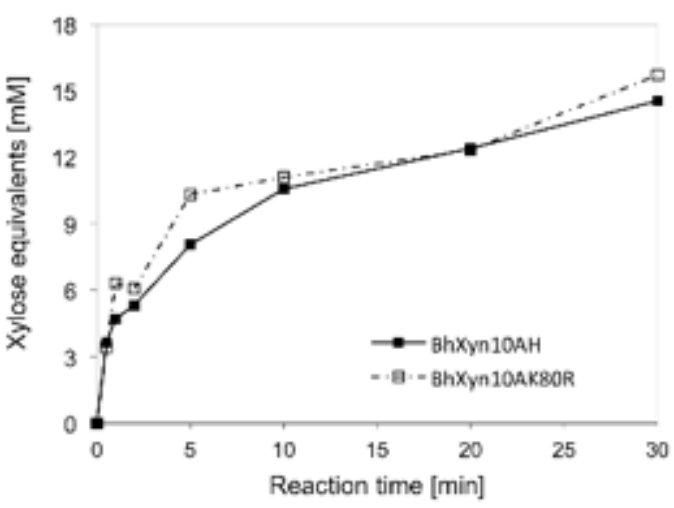

(B)

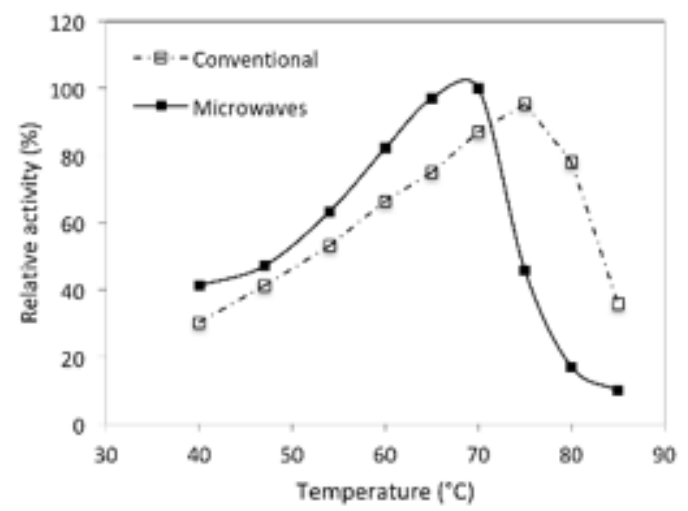

279 Based on the increasing tendency of the curves for the variants BhXyn10A and 280 BhXyn10AK80R, it could be suggested that the enzymes are not completely deactivated 281 after a half an hour of reaction under microwave radiation. Therefore, this could indicate 282 that the process is robust for this power input and reaction time, and even open the 283

Figure 2. Microwave assisted enzymatic reactions. (A) Time courses of birchwood xylan hydrolysis catalyzed by $B h \mathrm{Xyn} 10 \mathrm{~A}$ and variant $B h \mathrm{Xyn} 10 \mathrm{AK} 80 \mathrm{R}$. (B) Temperature profiles of the activity of $B h \mathrm{Xyn} 10 \mathrm{~A}$ both in conventional as well as in microwaves heated systems. possibility to try to increase both of the previously mentioned parameters, so as to increase yields. Overall, the mutant variant $B h \mathrm{Xyn} 10 \mathrm{AK} 80 \mathrm{R}$ performs slightly better than the wild type variant $B h X y n 10 \mathrm{~A}$; this observation is consistent with the higher activity of the mutant respect to the wild type reported in conventional conditions. ${ }^{1}$

The optimal temperature for $B h \mathrm{Xyn} 10 \mathrm{~A}$ under microwave heating is $68^{\circ} \mathrm{C}$ while under conventional heating is higher $75{ }^{\circ} \mathrm{C}$ (Figure 2B). However, the relative activity is significantly higher under microwaves irradiation in temperatures below $70^{\circ} \mathrm{C}$, while, in higher temperatures the relative activity is higher in conventional heating. These results shown that the enzyme is less tolerant to high temperatures in microwave than in conventional heating systems. 
BhXyn10AH

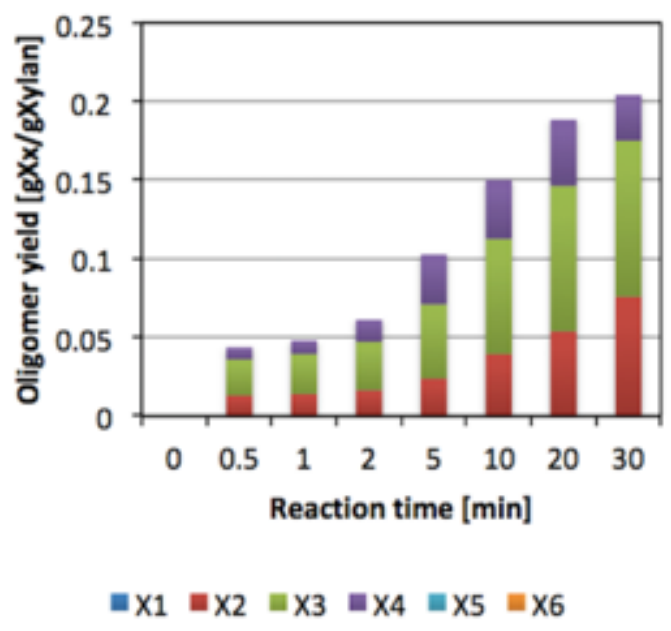

BhXYn10AK80R

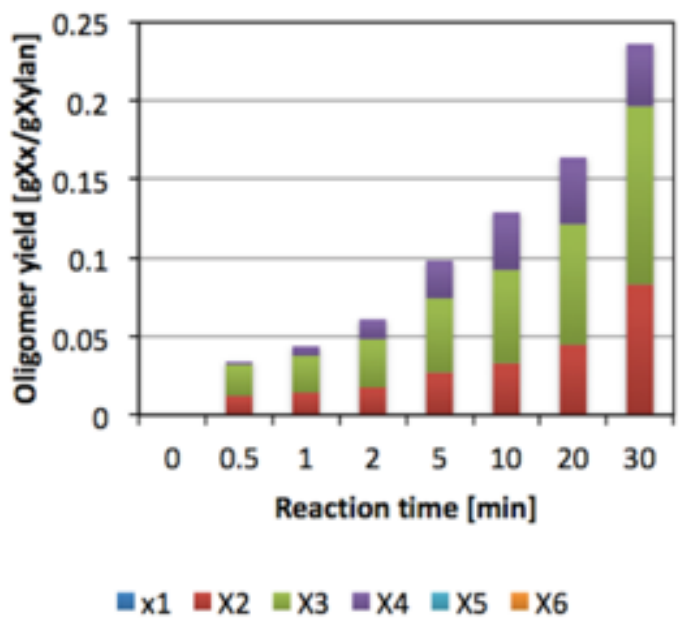

296 Figure 3. Composition profiles of the hydrolysates obtained by microwave-assisted enzymatic conversion of birchwood xylan. The analyzed oligomers are X1 (xylose), X2 (xylobiose), X3 (xylotriose), X4 (xylotetrose), X5 (xylopentose) and X6 (xylohexose).

Microwave-assisted reactions were initially carried out on birchwood xylan to observe the evolution of the profile throughout time. Only the enzyme BhXyn10A and $B h X y n 10 A K 80 \mathrm{R}$ were taken into account, since BhHXyn10A had been determined to be inactive under the studied experimental conditions. As a reference, the reactions were also performed on quinoa xylan for two-time values.

According to Figure 1, the enzymatic conversion of the four types of xylan by conventional heating produced a wide range of products, including a considerable proportion of xylose in all cases. Moreover, it can be seen that the variant $B h \mathrm{HXyn} 10 \mathrm{~A}$ was active in conventional heating conditions, even though its yield was significantly lower. On the other hand, microwave-assisted conversion of birchwood and quinoa

312 xylans did not produce any detectable amount of xylose in any studied time (Figure 4).

313 Also, it is important to note that the control mixtures which did not contain any enzyme and were only inactivated by microwave heating $\left(100^{\circ} \mathrm{C}\right)$ did not experience any detectable thermal hydrolysis. Therefore, the effect of spontaneous release of oligomers at high temperatures can be neglected. 
319 It is interesting to note that despite the marked improvement in the XOS yield obtained 320 by microwave-assisted heating in relation to traditional heating (approximately fivefold as it could be inferred from comparing Figure 1 and Figure 3), the reaction curves constructed based on the DNS assay show that the total concentration of products containing reducing end sugars is very similar in both cases. Further studies would need to be performed in order to approximate the composition profiles of xylosaccharides longer than six carbons in the obtained hydrolysates that might cause this observation. However, it should be highlighted that it was found that microwave-assisted heating produces a significant higher yield of XOS with a DP from 2 to 4 , which might be most adequate to specifically favor the development of beneficial microbes in the human gut. ${ }^{2}$

\subsection{Structural studies}

To find an explanation of the microwave effect in the reduced production of xylose, computational studies of the complex xylanase / ligands were performed. First, the complex BhXyn10AH / $\beta$-d-Xylp-(1-4)- $\beta$-d-Xylp-(1-4)- $\beta$-d-Xylp / $\beta$-d-Xylp-(1-4)- $\beta$-dXylp was build (Figure 4A) based on the crystallographic structures. The receptor is the xylanase from Bacillus halodurans (BhXyn10AH) (PDB: 2UWF), while the ligand was transferred from the co-crystallized structure of Streptomyces olivaceoviridis E-86 (PDB: 1ISX). The structure of the overall complex was energetically minimized as described in section 2.6.

BhXyn10AH has an $\alpha / \beta$-barrel 3D structure as all glycoside hydrolases from family GH10 (www.cazy.org) (Figure 4A). The catalytic site is a cleft containing at least four recognized subsites (Figure 4B): -2 and -1 in the glycone and +1 and +2 in the aglycone moiety. The catalytic amino acids, Glu265 and Glu159, were identified by superimposing the complex BhXyn10AH/ligands and the reference structure 1ISX. The enzyme/ligands model also allowed the prediction of amino acid/ligand interactions at each subsite (Figure 4C). Glycone subsites contain more hydrogen bonds than glycone subsites, which was also observed in other xylanases. ${ }^{5}$

Subsequently, the complex was subjected to molecular dynamics simulations to study the fluctuations in the interaction of each amino-acid residue with the ligands (Supplementary information S1 and S2). The root-mean-square-fluctuations (RMSF) shown that the 
amino acids in subsites +1 and +2 are more flexible than those located in subsites -2 and -1 (Figure 4D). Furthermore, the density of hydrogen bonds is higher in glycone than aglycone subsites, indicating a tighter ligand bond in glycone. All this suggest, that aglycone subsites are less specific and can bind two or one xylose units, resulting in the production of xylose as co-product. Thus, the microwave heating could affect the amino acid fluctuations in the aglycone subsites, favoring the interaction of two xylose subunits over one, reducing the xylose formation.

A

C
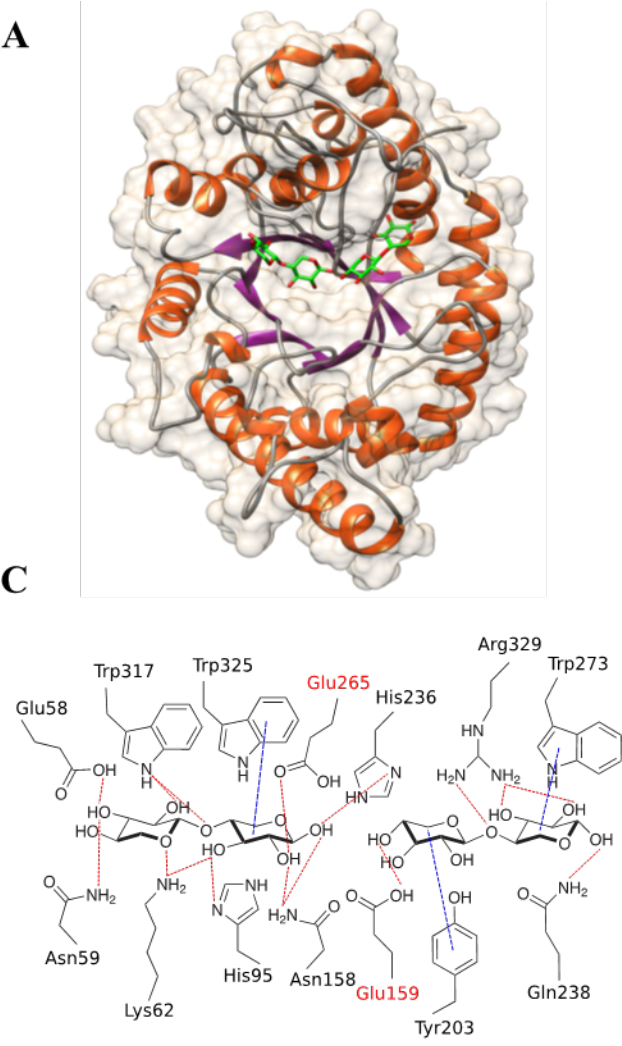

B

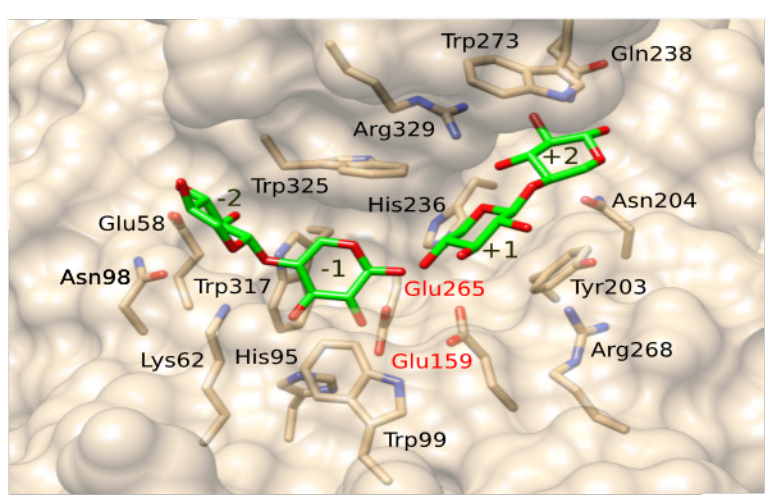

D

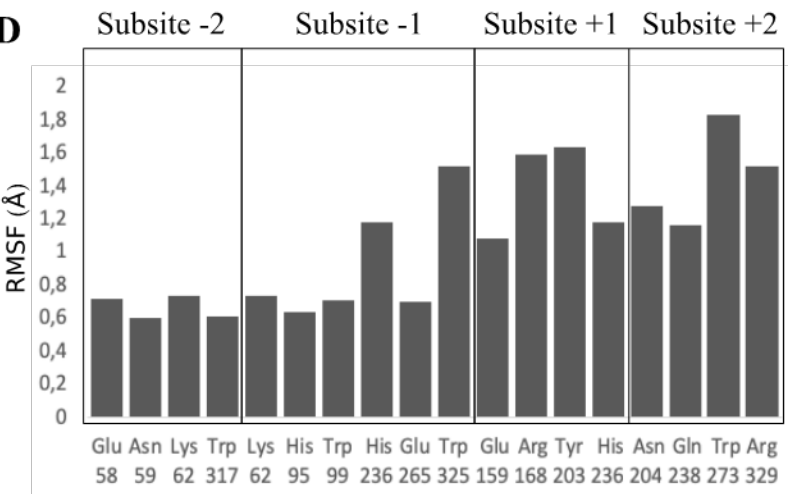

Figure 4. Complex enzyme/ligands BhXyn10AH / $\beta$-d-Xylp-(1-4)- $\beta$-d-Xylp-(1-4)- $\beta$-dXylp / $\beta$-d-Xylp-(1-4)- $\beta$-d-Xylp, modeled based on the crystallographic structure of the apoenzyme BhXyn10AH (2UWF). Ligands are represented in green (carbon atoms) and red (oxygen atoms). (A) Overall view of the enzyme / ligands model. (B) Predicted subsites and interactions between the active site amino-acids and ligands. Catalytic glutamates are labeled in red (Glu265 and Glu159). (C) Representation of the predicted interactions between enzyme and ligands. (D) Root-mean-square-fluctuations (RMSF), obtained by molecular dynamic simulations ( $50 \mathrm{~ns}$ ), of the amino-acids surrounding every subsite, some of them interact with the ligands. 


\section{Conclusion}

The microwave-assisted reaction markedly decreases the xylose content in the hydrolysates and significantly increases the yield of XOS, compared to conventional heating. Based on molecular dynamic simulations of BhXyn10A, we suggest that the microwave heating affect the amino acid fluctuations in the aglycone subsites reducing the xylose formation. The microwave-assisted heating yields a product that is significantly richer in xylooligosaccharides with a degree of polymerization that might selectively induce the growth of beneficial microorganisms in the human gut.

\section{Acknowledgements}

We thank the Center for Analysis and Synthesis (CAS), Lund University, for allowing us to use the microwave reactors.

\section{References}

1. R. Faryar, J. A. Linares-Pastén, P. Immerzeel, G. Mamo, M. Andersson, H. Stålbrand, B. Mattiasson and E. N. Karlsson, Food Bioprod. Process., 2015, 93, 1-10.

2. W. F. Broekaert, C. M. Courtin, K. Verbeke, T. Van de Wiele, W. Verstraete and J. A. Delcour, Crit. Rev. Food. Sci. Nutr., 2011, 51, 178-194.

3. J. A. Linares-Pasten, A. Aronsson and E. N. Karlsson, Curr. Protein. Pept. Sci., 2018, 19, 48-67.

4. E. Nordberg Karlsson, E. Schmitz, J. A. Linares-Pastén and P. Adlercreutz, Appl. Microbiol. Biotechnol., 2018, 102, 9081-9088.

5. A. Aronsson, F. Güler, M. V. Petoukhov, S. J. Crennell, D. I. Svergun, J. A. Linares-Pastén and E. Nordberg Karlsson, Biochim. Biophys. Acta Proteins Proteom., 2018, 1866, 292-306.

6. G. D. Yadav and S. V. Pawar, Bioresour. Technol, 2012, 109, 1-6.

7. A. L.-P. Javier, A. Maria and N. K. Eva, Curr. Biotechnol., 2014, 3, 26-44.

8. S. Patel and R. S. Gupta, International Journal of Systematic and Evolutionary Microbiology, 2020, 70, 406-438.

9. D. M. Salas-Veizaga, R. Villagomez, J. A. Linares-Pastén, C. Carrasco, M. T. Álvarez, P. Adlercreutz and E. Nordberg Karlsson, J. Agric. Food Chem., 2017, 65, 8663-8673.

10. A. Gil-Ramirez, D. M. Salas-Veizaga, C. Grey, E. N. Karlsson, I. RodriguezMeizoso and J. A. Linares-Pastén, Ind. Crops. Prod., 2018, 121, 54-65.

11. J. A. Linares-Pastén, P. Falck, K. Albasri, S. Kjellström, P. Adlercreutz, D. T. Logan and E. N. Karlsson, FEBS J., 2017, 284, 2019-2036.

12. S. Ramchuran, E. Nordberg Karlsson, S. Velut, L. de Maré, P. Hagander and O. Holst, Appl. Microbiol. Biotechnol., 2002, 60, 408-416. 
13. E. F. Pettersen, T. D. Goddard, C. C. Huang, G. S. Couch, D. M. Greenblatt, E. C. Meng and T. E. Ferrin, J. Comput. Chem., 2004, 25, 1605-1612.

14. E. Krieger and G. Vriend, Bioinformatics, 2014, 30, 2981-2982.

15. V. Case, D. Babin, J. Berryman, R. Betz, Q. Cai, D. Cerutti, T. Cheatham, T. Darden, R. Duke, H. Gohlke, A. Goetz, S. Gusarov, N. Homeyer, P. Janowski, J. Kaus, I. Kolossváry, A. Kovalenko, T. Lee, S. LeGrand, T. Luchko, R. Luo, B. Madej, K. Merz, F. Paesani, D. Roe, A. Roitberg, C. Sagui, R. Salomon-Ferrer, G. Seabra, C. Simmerling, W. Smith, J. Swails, R. Walker, J. Wang, R. Wolf, X. Wu and P. Kollman, Journal, 2014.

16. P. Mark and L. Nilsson, J. Phys. Chem. A, 2001, 105, 9954-9960.

17. T. Darden, D. York and L. Pedersen, The Journal of Chemical Physics 1993, 98, 10089-10092.

18. H. Berendsen, J. Postma, W. van Gunsteren, A. DiNola and J. R. Haak, J. Chem. Phys., 1984, 81, 3684-3690.

19. G. Yadav and S. Devendran, in White Biotechnology for Sustainable Chemistry eds. M. A. Coelho and B. Ribeiro, 2016, DOI: 10.1039/978178262408000052, ch. 4, pp. 52-103.

20. S. Laghari, M. Isa, A. Abdullah, A. Laghari and H. Saleem, IOSR J. Comput. Eng., 2014, 4, 14-28. 
Microwave-assisted xylanase reaction: the impact in the production of

441

442 prebiotic xylooligosaccharides

443

444

${ }^{1}$ Lund University, Division of Biotechnology, Department of Chemistry, Lund, Sweden

445

${ }^{2}$ Lund University, Centre for Analysis and Synthesis, Lund University, Lund, Sweden

446

447

${ }^{3}$ Technical University of Denmark, Department of Chemical and Biochemical Engineering, Copenhagen, Denmark

448

449

*Corresponding authors: J. A. Linares-Pastén

450

(e-mail: javier.linares_pasten@biotek.lu.se)

451

452

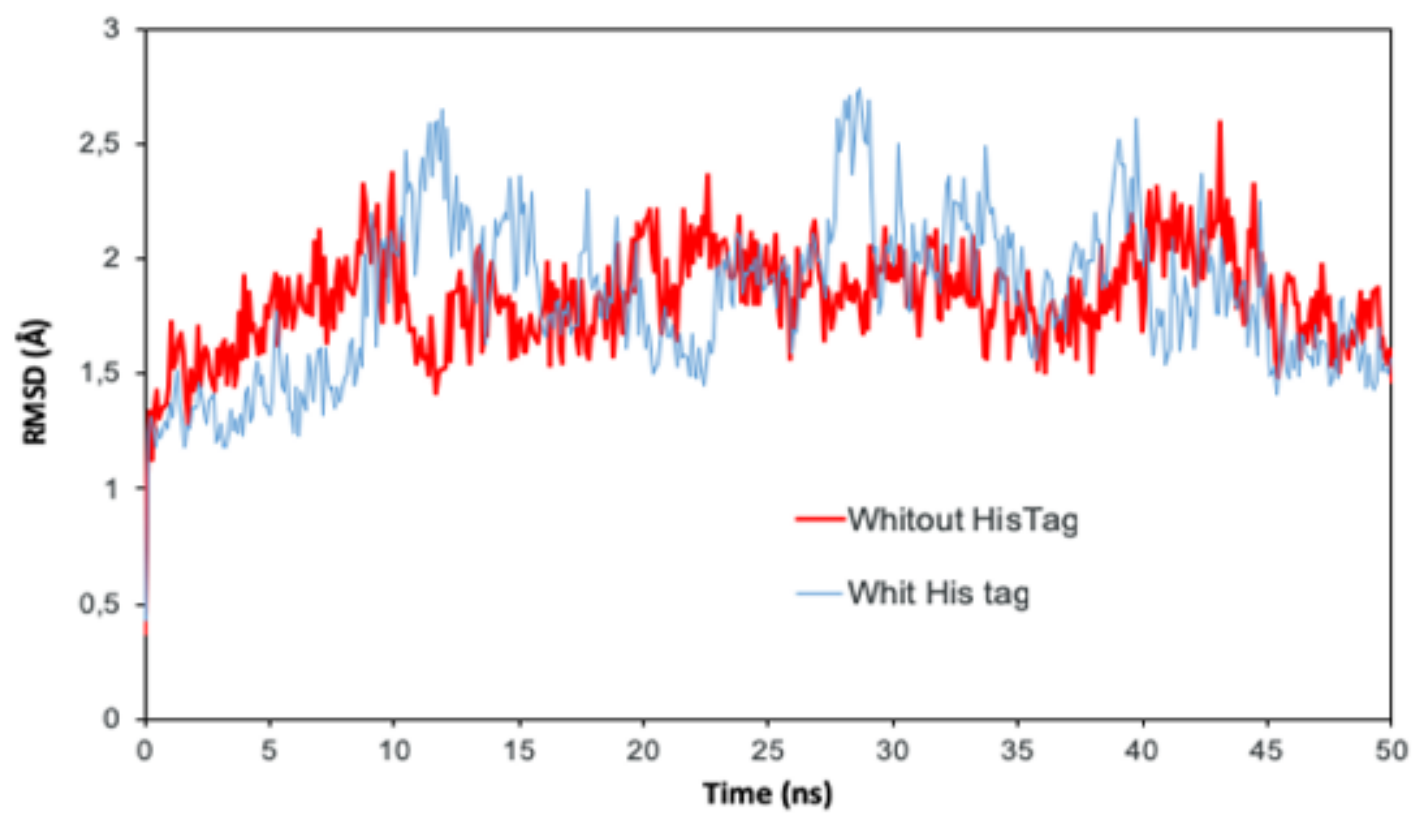

453

454 Figure 1S. Root-mean-square-deviations (RMSD) of the complex BhXyn10AH/ligands with and without 455 6x C-histidine tag. 


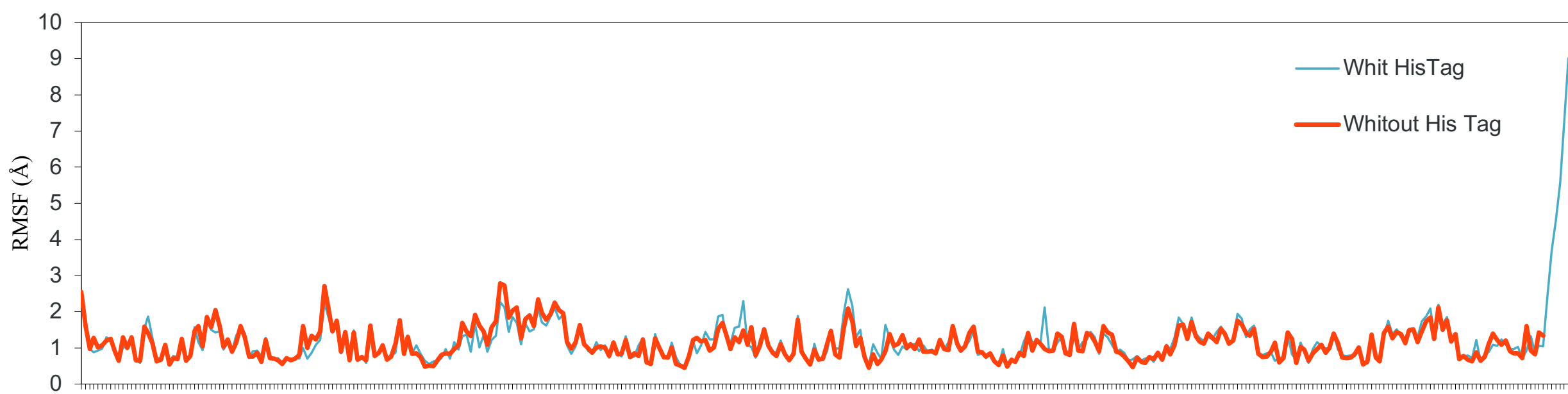

Aminoacids sequence 